\title{
LOS MAMÍFEROS SILVESTRES DE MILPA ALTA, DISTRITO FEDERAL: LISTA ACTUALIZADA Y CONSIDERACIONES PARA SU CONSERVACIÓN
}

\author{
Javier NAVARRO-FríAS ${ }^{1}$, Noé GONZÁLEZ-RUIZ1,2 \\ \& Sergio Ticul Álvarez-CASTAÑEDA ${ }^{3}$ \\ 1Laboratorio de Cordados Terrestres, Escuela Nacional de Ciencias Biológicas, \\ Instituto Politécnico Nacional. Prol. de Carpio y Plan de Ayala, Col. Santo Tomás. \\ Apartado Postal 42-186, 11340 México, D. F. MÉXICO \\ 2Universidad Autónoma Metropolitana, Departamento de Biología, Apartado Postal 55-535, \\ México 09340, D. F. MÉXICO \\ artilituratus@hotmail.com; artilituratus@yahoo.com \\ ${ }^{3}$ Centro de Investigaciones Biológicas del Noroeste, S. C. Mar Bermejo 195, 23000, \\ La Paz, Baja California Sur, MEXICO
}

\section{RESUMEN}

Las colectas realizadas durante dos años y la revisión de colecciones científicas confirmaron la presencia de 45 especies de mamíferos silvestres en la delegación Milpa Alta. Myotis thysanodes aztecus y Myotis volans amotus se registran por primera vez para el Distrito Federal. Cuatro especies con distribución en la Delegación están consideradas en alguna categoría de riesgo por el Gobierno Mexicano (Choeronycteris mexicana, Leptonycteris yerbabuenae, Cryptotis parva soricina y Romerolagus diazi). La décima parte de los géneros y la cuarta parte de las especies con distribución en la delegación son endémicas a México. Las especies de mamíferos presentes en la delegación Milpa Alta tienen afinidades con dos regiones zoogeográficas con diferentes prioridades de conservación. La parte norte de la delegación Milpa Alta, la más baja, está cubierta principalmente de matorral xerófilo y es la más afectada por las actividades humanas, como son la urbanización y los cultivos agrícolas. La parte sur, que corresponde a las zonas altas donde se ubica el bosque de coníferas, tiene mayor riqueza de especies y es la mejor conservada. Esta última debe ser considerada como refugio de fauna silvestre donde se puedan enfocar los programas para la conservación de mamíferos en el Distrito Federal.

Palabras clave: conservación, Distrito Federal, mamíferos silvestres, México, Milpa Alta.

\begin{abstract}
Field surveys and consults to mammal collections yield 45 native species of mammals for the Milpa Alta Delegation. Myotis thysanodes aztecus and Myotis volans amotus are recorded for the first time for the Federal District. Four species are included as threatened by the Mexican Government (Choeronycteris mexicana, Leptonycteris yerbabuenae, Cryptotis parva soricina y Romerolagus diazi). Ten percent of the genera and one fourth of the species with distribution in this region are endemic to Mexico. Mammals present in the Milpa Alta Delegation have affinity to two biogeographic regions with different conservation priorities. The northern part, which is the most affected by human activities, includes the lowlands with xerophytic scrub vegetation. The southern area, the best preserved and with
\end{abstract}


the highest species richness, includes the highlands with coniferous forest. This region should be considered a wildlife refuge and the focus for fauna conservation programs on the Federal District.

Key words: annotated checklist, conservation, Federal District, mammals, Milpa Alta .

\section{INTRODUCCIÓN}

En el Distrito Federal, el crecimiento desenfrenado de la población humana ha tenido como consecuencia el aumento de los asentamientos humanos, y por lo tanto el incremento del deterioro ambiental, lo que ha repercutido en la reducción cada vez mayor de los espacios que pueden albergar a la flora y fauna silvestres. Ante estos hechos, la fauna silvestre en general enfrenta serios problemas de conservación, principalmente por la drástica reducción del hábitat. Esta situación ha provocado que algunas especies de mamíferos se hayan extirpado (e. i. Dipodomys phillipsii y Lepus callotis), mientras que un número más reducido ha logrado sobrevivir en los suburbios o en algunos pocos refugios dentro de la metrópoli (e.i. Cratogeomys merriami, Sciurus aureogaster y Peromyscus truei), pero con un futuro incierto.

Son pocas las áreas dentro del Distrito Federal que aún conservan la vegetación original y por lo tanto, que se puedan considerar como recintos biológicos donde la fauna silvestre actualmente sobreviva. La delegación Milpa Alta es prácticamente la única zona del Distrito Federal que todavía conserva áreas en condiciones adecuadas para albergar a varias especies de mamíferos silvestres del Valle de México. Este hecho se debe a la combinación de diferentes factores: (1) tiene el menor número de habitantes (aprox. 156,000) entre las delegaciones del Distrito Federal, (2) la actividad económica de la zona se enfoca principalmente a la agricultura, (3) el uso del suelo es ejidal, lo que hasta hace unos años limitaba el crecimiento de la mancha urbana en esa dirección y (4) la actividad industrial es de las más bajas del Valle de México. Esta amalgama de condiciones hasta el momento le confiere a la delegación Milpa Alta una mayor superficie de áreas relativamente bien conservadas, aunque la vegetación original se encuentra en manchones, lo que se refleja en una distribución fragmentada de las especies de mamíferos.

La delegación Milpa Alta tiene en la parte sur una zona boscosa, que se considera parte del corredor biológico Chichinautzin, que es prácticamente continuo desde la región de Zempoala (Morelos-Estado de México) hasta la de Zoquiapan (Estado de México), incluyendo la ladera oeste de los volcanes del Popocatépetl e Iztacíhuatl. Este corredor biológico es de gran importancia, debido a que es la única conexión actual que puede permitir la migración y el intercambio genético de especies silvestres en la parte central del Eje Volcánico Transversal. La mastofauna de esta región ha sido evaluada en algunas de sus partes como en las Lagunas de Zempoala 
(Ramírez-Pulido 1969), Sierra del Ajusco (Aranda et al. 1980) en su parte oeste y en la estación biológica de Zoquiapan (Blanco et al. 1981) en la este. En la región sur de este corredor, sólo se tiene información de la parte baja dentro del estado de Morelos (Álvarez-Castañeda 1996), sin que a la fecha se tenga información detallada de la parte alta del corredor y de la ladera sur del Valle de México, que es la región más expuesta a la presión humana.

Es notable el hecho de que a pesar de la importancia del Distrito Federal como centro económico, político y académico del país, los estudios que se han realizado sobre mamíferos silvestres sean muy pocos, y particularmente sobre la delegación Milpa Alta. El registro más antiguo del que se tiene conocimiento es el de Peromyscus maniculatus (Hooper 1947) y los que se incluyen dentro de la obra "Los Mamíferos silvestres del Valle de México" (Villa-Ramírez 1953) en el que se registra a Leptonycteris yerbabuenae y Glossophaga soricina. Entre otros trabajos de la zona están los de Velázquez et al. (1996) sobre Romerolagus diazi y González-Romero (1981) sobre roedores plaga.

Es inminente que la Ciudad de México seguirá en proceso de expansión hacia el sur, por lo que la delegación Milpa Alta será un objetivo de desarrollo urbano a mediano plazo. Por lo tanto, es necesario conocer la fauna de mamíferos de la Delegación para estimar el estado actual de las especies que habitan el corredor biológico y así, en un futuro próximo, poder implementar programas de conservación y protección de la región y sus especies.

\section{MATERIAL Y MÉTODOS}

\section{Área de estudio}

La delegación Milpa Alta se ubica al sureste del Distrito Federal (19 $03^{\circ}, 19^{\circ} 13^{\text {' }}$ de latitud norte y $98^{\circ} 57^{\prime}, 99^{\circ} 10^{\prime}$ de longitud oeste). Tiene una superficie de $279 \mathrm{~km}^{2}$ y está enclavada en la Sierra del Chichinautzin, por lo que su relieve es abrupto, variando desde los $2240 \mathrm{~m}$ hasta los $3000 \mathrm{~m}$ (Reyes 1989; Fig. 1). A pesar de la corta extensión de la Delegación, presenta cuatro climas que van desde templado subhúmedo con lluvias en verano $\mathrm{C}(\mathrm{w} 2)$ hasta el semifrío subhúmedo con lluvias en verano $\mathrm{C}(\mathrm{E})(\mathrm{w} 2)$, este último cubre la mayor porción de la delegación $(69.6 \%)$ y se encuentra en toda su parte centro-sur (INEGI, 1997).

En la delegación Milpa Alta existen principalmente tres asociaciones vegetales naturales, matorral xerófilo, bosque de pino y bosque de oyamel, además de otras que son inducidas (áreas de cultivo). El bosque de pino se desarrolla en casi toda la parte sur de la delegación, de los 2300 msnm hasta sus partes más altas. Las especie vegetales predominantes son Pinus leiophylla, $P$. montezumae y $P$. hartweggii, aunque en ocasiones entran en la composición de este bosque otras 
especies de Pinus, así como algunas de Quercus, Abies, Salix, y Buddleia (Rzedowski \& Rzedowski 1979). El bosque de oyamel se presenta sólo en pequeños manchones del sureste, generalmente en altitudes entre 2700 a 3000 m; la especie dominante y con frecuencia exclusiva en el estrato superior es Abies religiosa (Rzedowski 1975). En la parte norte y centro, la vegetación natural era matorral xerófilo (Rzedowski \& Rzedowski 1979) pero esta región fue substituida por zonas urbanas y áreas de cultivo principalmente (Fig. 1). Los cultivos agrícolas que más comúnmente se siembran son el nopal (Opuntia sp.), maíz (Zea maiz), haba (Vicia faba) y frijol (Phaseolus sp.), aunque en ocasiones se encuentran intercalados con pequeños manchones de vegetación natural de matorral (Senecio sp.) y de bosque de Pinus leiophylla.

Durante 1999 y 2000 se realizaron 18 visitas al campo para registrar los mamíferos silvestres que habitan la región mediante métodos directos e indirectos. Para la colecta de murciélagos, se utilizaron redes de nylon japonesas de 6 a $12 \mathrm{~m}$ de longitud, que fueron colocadas antes del anochecer atravesando los arroyos, en la vegetación abierta y en muchos casos en bosques con vegetación densa, revisándose de manera periódica durante la noche. Para los mamíferos no voladores se utilizaron trampas de golpe tipo "Victor" y "Museum Special"; y "Sherman". Todas las trampas se cebaron con avena y se colocaron en transectos al anochecer, revisándolas repetidamente durante la noche. En el caso de los mamíferos de talla mediana,

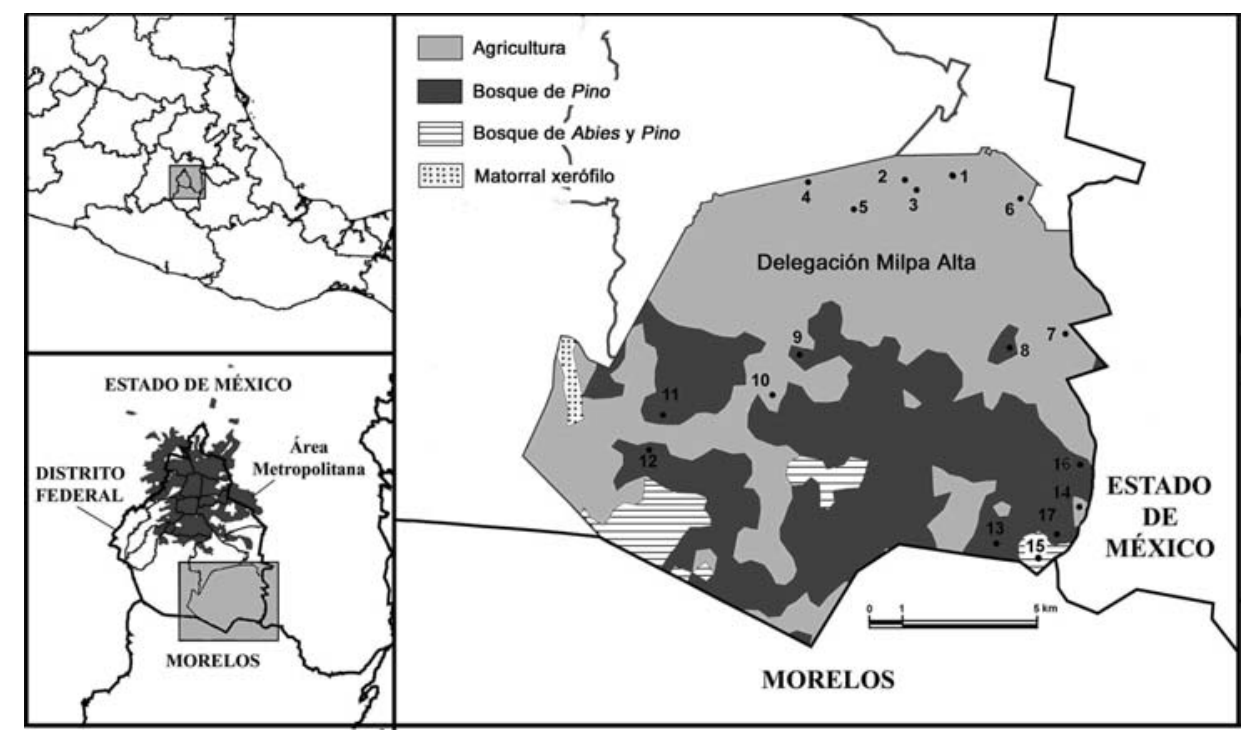

Figura 1

Ubicación geográfica y tipos de vegetación de la delegación Milpa Alta, Distrito Federal. Los puntos numerados indican las localidades de colecta (ver Cuadro 1). 


\section{Cuadro 1}

Especies por localidad de colecta en la delegación Milpa Alta.

\begin{tabular}{|c|c|c|c|c|c|c|c|c|c|c|c|c|c|c|c|c|c|}
\hline \multirow[b]{2}{*}{ Especie } & \multicolumn{17}{|c|}{ Localidades de colecta } \\
\hline & 1 & 2 & 3 & 4 & 5 & 6 & 7 & 8 & 9 & 10 & 11 & 12 & 13 & 14 & 15 & 16 & 17 \\
\hline Didelphis virginiana & $\mathrm{X}$ & & & & & & & & & & & & & $\mathrm{X}$ & $\mathrm{X}$ & & \\
\hline Cryptotis parva & & & & & & & & & & & & & & & & $\mathrm{X}$ & \\
\hline Choeronycteris mexicana & & & $\mathrm{X}$ & & & & & & & & & & & & & & \\
\hline Myotis californicus & & & & & & & & & & & & & & & $\mathrm{X}$ & & \\
\hline Myotis thysanodes & & & & & & & & & & & & & & & $\mathrm{X}$ & & \\
\hline Myotis velifer & & & & & & & & & & & & & & & $\mathrm{X}$ & & \\
\hline Myotis volans & & & & & & & & & & & & & & & $\mathrm{X}$ & & \\
\hline Eptesicus fuscus & & & & & & & & & & & & & & & $\mathrm{X}$ & & \\
\hline Lasiurus blossevillii & & & & & & & & & & & & & & & $\mathrm{X}$ & & \\
\hline Lasiurus cinereus & & & & & & & & & & & & & & & $\mathrm{X}$ & & \\
\hline Corynorhinus mexicanus & & & & & & & & & & & & & & & $\mathrm{X}$ & & \\
\hline Corynorhinus town sendi & & & & $\mathrm{X}$ & & & & & & & & & & & & & \\
\hline Tadarida brasiliensis & & & & & $\mathrm{X}$ & & & & & & & & & & & & \\
\hline Romerolagus diazi & & & & & & & & & $\mathrm{X}$ & & & & & & & & \\
\hline Sylvilagus floridanus & & & & & & & & & & $\mathrm{X}$ & & & & & & & \\
\hline Sciurus aureogaster & & & $\mathrm{X}$ & & & & & & & & & & & & & & \\
\hline Liomys irroratus alleni & $\mathrm{X}$ & $\mathrm{X}$ & $X$ & & & & $\mathrm{X}$ & & & & & & & & & & \\
\hline Reithrodontomys chrysopsis & & & & & & & & & & & & & $\mathrm{X}$ & & $\mathrm{X}$ & & $\mathrm{X}$ \\
\hline Reithrodontomys fulvescens & $\mathrm{X}$ & $X$ & $\mathrm{X}$ & & & & & & & & & & & & & & \\
\hline Reithrodontomys megalotis & & & & & & & $\mathrm{X}$ & & & & & $\mathrm{X}$ & & & $\mathrm{X}$ & & \\
\hline Reithrodontomys sumichrasti & & & & & & & & & & & & & & & $\mathrm{X}$ & & \\
\hline Peromyscus difficilis amplus & & & $\mathrm{X}$ & & & & & & & & & & & & & & \\
\hline Peromyscus difficilis felipensis & & & & & & & & & & & & $\mathrm{X}$ & $\mathrm{X}$ & & $\mathrm{X}$ & $\mathrm{X}$ & \\
\hline Peromyscus gratus & $\mathrm{X}$ & & $\mathrm{X}$ & & & & & & & & & & & & & & \\
\hline Peromyscus levipes & & & $X$ & & & & & & & & & & & & & & \\
\hline Peromyscus maniculatus fulvus & & $\mathrm{X}$ & $\mathrm{X}$ & & & & & & & & & & & & & & \\
\hline $\begin{array}{l}\text { Peromyscus maniculatus } \\
\text { labecula }\end{array}$ & & & & & & & & $\mathrm{X}$ & & & & & & & & & \\
\hline Peromyscus melanotis & & & & & & & & & & & & $\mathrm{X}$ & $\mathrm{X}$ & & $\mathrm{X}$ & & \\
\hline Baiomys taylori & & & $\mathrm{X}$ & & & $\mathrm{X}$ & & & & & & & & & & & \\
\hline Neotomodon alstoni & & & & & & & & & & & $\mathrm{X}$ & $\mathrm{X}$ & $\mathrm{X}$ & & $\mathrm{X}$ & $X$ & \\
\hline Neotoma mexicana & & & & & & & & & & & & & & & $\mathrm{X}$ & & \\
\hline Microtus mexicanus & & & & & & & & & & & $\mathrm{X}$ & & & & & & \\
\hline Spilogale putorius & & & & & & & & & & & & & & $\mathrm{X}$ & & & \\
\hline Conepatus mesoleucus & & & & & & & & & & & & & & & $\mathrm{X}$ & & \\
\hline Tipo de Vegetación & $\mathrm{AC}$ & $\mathrm{AC}$ & MX & $\mathrm{AC}$ & $\mathbf{A C}$ & $\mathrm{AC}$ & $\mathrm{AC}$ & BP & BP & $\mathrm{AC}$ & BP & BP & BP & $\mathbf{A C}$ & BAP & BP & $3 P$ \\
\hline
\end{tabular}

Las abreviaciones del tipo de vegetación corresponden a: AC, área de cultivo (en todos los casos la vegetación original era matorral xerófilo); MX, matorral xerófilo; BP, bosque de Pino; BAP, bosque de Abies y Pino. El número de las localidades se corresponde con la Figura 1: (1) Volcán Teutli, 1 km N, 2 km W Tecomitl; (2) Volcán Teutli, $3.0 \mathrm{~km} \mathrm{~N}, 1.0 \mathrm{~km}$ W Milpa Alta; (3) Volcán Teutli, $3.2 \mathrm{~km}$ N, $1.2 \mathrm{~km}$ W Milpa Alta; (4) 3 km NW San Pedro Actopan; (5) San Pedro Actopan; (6) 1 km E Tecomitl; (7) 1 km S, 3 km E Santa Ana Tlacotenco; (8) 2 km S, Santa Ana Tlacotenco; (9) 4.5 km S, 0.5 km W San Pablo Ostotepec; (10) 6.5 km S, 1 km W San Pablo Ostotepec; (11) 7 km S, 3 km W San Salvador Cuahutenco; (12) 9 km S, 4 km W San Salvador Cuahutenco; (13) 10 km S Santa Ana Tlacotenco; (14) Volcán Pelagatos, 9 km S, 3.2 km E Santa Ana Tlacotenco; (15) La Quinta, 11 km S, 2 km E Santa Ana Tlacotenco; (16) 4.5 km S, 3 km E Santa Ana Tlacotenco; (17) $9.5 \mathrm{~km} \mathrm{~S}, 2.5 \mathrm{~km}$ E Santa Ana Tlacotenco. 
se utilizaron trampas para ejemplares vivos del tipo "Tomahawk". Los registros indirectos consistieron en avistamientos, colecta de cadáveres, excretas y algunas egagrópilas aisladas, que posteriormente fueron analizadas. Un grupo de ejemplares representativos de cada especie se prepararon según las técnicas convencionales en piel y cráneo (Hall 1981) y se depositaron en la colección mastozoológica de la Escuela Nacional de Ciencias Biológicas del Instituto Politécnico Nacional (ENCB).

Para identificar a los ejemplares se consultaron diferentes claves dicotómicas (Álvarez et al. 1994, Hall 1981, Hooper 1952). Para el arreglo taxonómico de las especies se siguió a Hall (1981) y para la nomenclatura se tomaron las recomendaciones de Ramírez-Pulido et al. (2005), con excepción de Leptonycteris curasoae que se conservó como L. yerbabuenae de acuerdo a los criterios de Álvarez \& Álvarez-Castañeda (1996). En la mayoría de los casos el material colectado, así como el procedente de las excretas y egagrópilas, se comparó con ejemplares previamente identificado de colecciones mastozoológicas.

Para complementar los datos obtenidos durante nuestras colectas, se consultaron las colecciones mastozoológicas del Instituto de Biología de la Universidad Nacional Autónoma de México (IB-UNAM), la del Museo de Zoología "Alfonso L. Herrera" de la Facultad de Ciencias de la Universidad Nacional Autónoma de México (MZFC) y la de la Universidad Autónoma Metropolitana Unidad Iztapalapa (UAMI). De la etiqueta de cada uno de los ejemplares, se tomaron, tal y como están consignados, el sexo, los datos reproductivos, la localidad de colecta y fecha de colecta.

\section{RESULTADOS}

Durante el trabajo de campo se capturaron 323 ejemplares que corresponden a 34 especies y se obtuvieron registros indirectos (huellas, excretas y observaciones) de nueve especies adicionales. De las colecciones científicas se registraron 45 ejemplares de nueve especies y de la revisión bibliográfica se obtuvieron seis especies. En conjunto, en la delegación Milpa Alta se presentan 45 especies que pertenecen a 34 géneros, 14 familias y 8 órdenes. El orden mejor representado es Rodentia con 19 especies (42.2\%), seguido de Chiroptera 13 (28.8\%), Carnivora 7 (15.5\%), Lagomorpha 2 (4.4\%), Insectivora 2 (4.4\%) y Didelphimorphia, Xenarthra y Artyodactila, cada uno con una especie (4.7\%). E1 $8.8 \%$ de las especies de Milpa Alta está incluido dentro de la Norma Oficial Mexicana (SEMARNAT 2002). De este porcentaje, dos especies están catalogadas como amenazadas (Choeronycteris mexicana y Leptonycteris yerbabuenae), una bajo protección especial (Cryptotis parva soricina) y una más en peligro de extinción (Romerolagus diazi). 


\section{TRATADO TAXONÓMICO}

\section{Orden Didelphimorphia \\ Familia Didelphidae \\ Didelphis virginiana californica Bennett, 1833}

Ejemplares examinados, 3: Volcán Teutli, 1 km N, 2 km W Tecomitl, 2400 m (ENCB); Volcán Pelagatos, 9 km S, 3.2 km E Santa Ana Tlacotenco, 2900 m (ENCB); La Quinta 11 km S, 2 km E Santa Ana Tlacotenco, 2850 m (ENCB).

Los ejemplares se encontraron muertos en avanzado estado de descomposición por lo que no fue posible determinar el sexo. El ejemplar del volcán Teutli se halló dentro de una pequeña cueva que se encuentra a no más de $200 \mathrm{~m}$ de la zona habitada del pueblo de Tecomitl, los otros dos se encontraron dentro de piletas secas que se ubican entre bosque de pino y bosque de oyamel. La especie había sido previamente registrada para este tipo de vegetación (Ramírez-Pulido 1969) para la zona de Zempoala, en Morelos.

\section{Orden Soricomorpha \\ Familia Soricidae \\ Sorex oreopolus Merriam, 1892}

Ejemplares examinados, 1: Chicomostoc Cerro Teutli, 2.8 mi. NNW Milpa Alta, 2620 m (IBUNAM).

Este ejemplar fue colectado en 1948; la especie no se ha registrado recientemente en las cercanías a la delegación; sin embargo, es muy probable que todavía se le encuentre en la región, debido a que su hábitat como bosque de pino y áreas húmedas con musgo, zonas riparias y praderas (Hennings \& Hoffmann 1977) aún no ha desaparecido.

\section{Cryptotis parva soricina (Merriam, 1895)}

Ejemplares examinados, 2: $4.5 \mathrm{~km} \mathrm{~S}, 3 \mathrm{~km}$ E Santa Ana Tlacotenco, $2800 \mathrm{~m}$ (ENCB).

Los ejemplares fueron recuperados de una egagrópila, presumiblemente de lechuza (Tyto $a l b a)$, que se encontraba dentro de un pastizal amacollado intercalado con cultivos de maíz.

\section{Orden Chiroptera Familia Phyllostomidae} Glossophaga soricina handleyi Webster y Jones, 1980

Ejemplares examinados, 15: Chicomostoc Cerro Teutli, 2.8 Millas NNW Milpa Alta, $2620 \mathrm{~m}$ (IB-UNAM).

Todos los ejemplares son hembras y tienen un peso promedio de $7.4 \mathrm{~g}$ (6.5-8.2). VillaRamírez (1953) identifica inicialmente a estos especimenes como Glossophaga soricina morenoi; posteriormente son asignados a dos diferentes especies G. soricina leachii y $G$. morenoi (Villa-Ramírez 1967). En las revisiones ulteriores realizadas por Sánchez et al. (1989) y Webster (1993) son re-identificados como G. s. handleyi, asignación que nosotros compartimos. Por lo anterior, el registro de G. morenoi no es válido para la delegación Milpa Alta. 


\section{Choeronycteris mexicana Tschudi, 1844}

Ejemplares examinados, 1: Volcán Teutli, $3.2 \mathrm{~km} \mathrm{N,} 1.2 \mathrm{~km}$ W Milpa Alta, $2550 \mathrm{~m}$ (ENCB). Esta hembra se colectó en agosto sin signos aparentes de reproducción. Fue capturada dentro de una pequeña cueva en la que había regular oscuridad, aunque también se le ha colectado en refugios con oscuridad total (Villa-Ramírez 1967). Previamente se había colectado en áreas próximas de selva baja caducifolia, como en las Grutas de la Estrella, $6 \mathrm{~km} \mathrm{S,} 4 \mathrm{~km}$ E Tonatico y Barranca de los Ídolos, en el Estado de México (González-Ruiz et al. 2004, Villa 1967).

\section{Leptonycteris yerbabuenae Villa-Ramírez y Martínez, 1940}

Ejemplares examinados, 12: Chicomostoc Cerro Teutli, 2.8 mi. NNW Milpa Alta, $2620 \mathrm{~m}$ (IBUNAM).

El peso promedio de los ejemplares es de $20.4 \mathrm{~g}$ (17.5-27.0). Los ejemplares fueron inicialmente identificados como Leptonycteris nivalis (Villa-Ramírez 1953). En la revisión de los mamíferos de México (Villa-Ramírez 1967) se reconsidera la determinación y se asignan a L. nivalis y $L$. yerbabuenae. Estudios recientes incluyen a todos estos ejemplares como L. yerbabuenae (RamírezPulido \& Álvarez 1972, Arita \& Humphrey 1988, Sánchez et al. 1989).

\section{Familia Vespertilionidae}

Myotis californicus mexicanus (Saussure, 1860)

Ejemplares examinados, 15: La Quinta, $11 \mathrm{~km} \mathrm{S,} 2 \mathrm{~km}$ E Santa Ana Tlacotenco, $2850 \mathrm{~m}$ (ENCB).

Se colectaron seis hembras. En mayo se obtuvo una preñada con un embrión de $14 \mathrm{~mm}$ de longitud y otra estaba lactante. Las hembras restantes se colectaron en febrero, marzo y diciembre, sin signos aparentes de reproducción. Nueve machos colectados en mayo y julio, presentaron una longitud promedio de los testículos de $1.6 \mathrm{~mm}$ (1.0-2.0). Dado que los ejemplares se colectaron durante casi todo el año (febrero, marzo, mayo, julio y diciembre), se puede suponer que la especie tiene poblaciones permanentes en la región.

Los especimenes se colectaron en una red colocada sobre una "pileta" que capta el agua de un manantial, dentro de una cañada con bosque de oyamel y pino. En la misma red también se capturaron ejemplares de: Myotis thysanodes, M. velifer, M. volans, Eptesicus fuscus, Lasiurus blossevillii, L. cinereus y Corynorhinus mexicanus.

\section{Myotis thysanodes aztecus Miller y G. M. Allen, 1928}

Ejemplares examinados, 5: La Quinta, $11 \mathrm{~km} \mathrm{S,} 2$ km E Santa Ana Tlacotenco, $2850 \mathrm{~m}$ (ENCB). Estos ejemplares constituyen el primer registro de la especie para el Distrito Federal. La localidad más cercana de donde se había registrado la especie es Tlayacapan, Morelos (Álvarez-Castañeda 1996). Todos los ejemplares examinados son machos capturados durante mayo y julio. La longitud media de los testículos fue $3.4 \mathrm{~mm}(2.0-5.0)$ y el peso medio $7.0 \mathrm{~g}$ (5.9-7.8). Estos especimenes se colectaron en las mismas condiciones que Myotis californicus.

\section{Myotis velifer velifer (J. A. Allen, 1890)}

Ejemplares examinados, 22: La Quinta, $11 \mathrm{~km} \mathrm{S,} 2 \mathrm{~km}$ E Santa Ana Tlacotenco, $2850 \mathrm{~m}$ (ENCB). 
Dos hembras colectadas en julio y en agosto no mostraron signos de actividad reproductiva. Once machos, capturados durante mayo con una longitud testicular media de $3.7 \mathrm{~mm}$ (2.0-5.0) y nueve en julio, agosto y octubre con una de $5.0 \mathrm{~mm}$ (3.0-8.0). El aumento testicular observado hacia el invierno, coincide con lo registrado para ejemplares de Michoacán (Villalpando \& Álvarez 2000). Sin embargo, esta especie como otras del género Myotis puede copular antes del invierno (Fitch et al. 1981), datos similares se obtuvieron de ejemplares de Morelos (ÁlvarezCastañeda 1996). Esta especie solamente se colectó en primavera y verano, por lo que es posible que realice movimientos altitudinales según la estación del año (Villa-Ramírez 1967).

\section{Myotis volans amotus Miller, 1914}

Ejemplares examinados, 37: La Quinta, $11 \mathrm{~km} \mathrm{~S}, 2 \mathrm{~km}$ E Santa Ana Tlacotenco, $2850 \mathrm{~m}$ (ENCB).

Esta especie no se había registrado con anterioridad para el Distrito Federal. El registro más cercano era de $1.5 \mathrm{~km}$ al NE San Juan Tlacotenco, Morelos (Guerrero-Enríquez et al. 1996) pero estos especímenes son muy diferentes en las medidas corporales. Al revisar los ejemplares de Morelos encontramos que corresponden a Myotis californicus de acuerdo con las características dadas por Miller \& Allen (1928). Por esta razón el registro de Morelos queda invalidado; entonces el registro más cercano es de la ladera occidental del Volcán Popocatépetl en el Estado de México (Ceballos \& Galindo 1984).

Se colectaron 12 machos en julio con una longitud media testicular de $4.0 \mathrm{~mm}(3.0-5.0)$ y 10 hembras en mayo, cuatro de éstas se encontraban gestando cada una con un embrión (longitud promedio $17.0 \mathrm{~mm}$; extensión 15.0-19.0); una presentó dos embriones y cinco estaban lactando; sólo una de ellas transportaba su cría sujeta al pezón. Es posible que la época de crianza de esta especie sea únicamente durante la primavera, pues también se han encontrado hembras preñadas durante mayo y julio en otros estados de la república (Baker \& Phillips 1965, Watkins et al. 1972, Ramírez-Pulido et al. 1980). Todos los ejemplares fueron capturados en las mismas condiciones que Myotis californicus.

\section{Eptesicus fuscus miradorensis (H. Allen, 1866)}

Ejemplares examinados, 23: La Quinta, $11 \mathrm{~km} \mathrm{~S}, 2 \mathrm{~km}$ E Santa Ana Tlacotenco, $2850 \mathrm{~m}$ (ENCB).

La única hembra colectada fue en febrero y no tenía signos aparentes de reproducción. El resto de los especimenes son machos, siete colectados en febrero con una longitud testicular media de $3.4 \mathrm{~mm}(3.0-5.0)$ y 12 en marzo y mayo $(6.3 ; 3.0-10.0 \mathrm{~mm})$. La especie se asocia a las partes altas de México y previamente había sido colectada de la zona boscosa de las lagunas de Zempoala (Davis 1944, Davis \& Russell 1954). Esta especie fue colectada en las mismas condiciones que Myotis californicus.

\section{Lasiurus blossevillii frantzi (Peters, 1871)}

Ejemplares examinados, 8: La Quinta, $11 \mathrm{~km} \mathrm{S,} 2$ km E Santa Ana Tlacotenco, $2850 \mathrm{~m}$ (ENCB).

Todos los ejemplares son machos colectados en diciembre, febrero y marzo. La longitud media de los testículos es de $3.5 \mathrm{~mm}$ (3.0-4.0). Todos se encontraron sólo en la temporada fría 
por lo que es posible que esta zona sea un refugio invernal, debido a que esta especie se considera como migratoria (Shump \& Shump 1982a; Bogan 1999). Los ejemplares se colectaron en las mismas condiciones que Myotis californicus, sin embargo, no se colectó junto con M. thysanodes, M. velifer ni Corynorhinus mexicanus.

\section{Lasiurus cinereus cinereus (Palisot de Beauvois, 1796)}

Ejemplares examinados, 4: La Quinta, $11 \mathrm{~km} \mathrm{S,} 2 \mathrm{~km} \mathrm{E} \mathrm{Santa} \mathrm{Ana} \mathrm{Tlacotenco,} 2850 \mathrm{~m}$ (ENCB).

Los especimenes son machos capturados en mayo y diciembre, con una longitud testicular media de $4.0 \mathrm{~mm}$ (3.0-5.0). Hall \& Villa (1949) mencionan que la especie probablemente inverna en México, lo que concuerda con el hecho de haberlos capturado sólo en el periodo frío del año (Shump \& Shump 1982b, González-Ruiz et al. 2004). Esta especie se colectó en las mismas condiciones que Myotis californicus.

\section{Corynorhinus mexicanus G. M. Allen, 1916}

Ejemplares examinados, 9: La Quinta, $11 \mathrm{~km} \mathrm{S,} 2 \mathrm{~km}$ E Santa Ana Tlacotenco, $2850 \mathrm{~m}$ (ENCB).

Siete hembras y dos machos fueron colectadas durante mayo, todas las hembras estaban lactando y uno de los machos era juvenil. Debido a que la localidad de La Quinta se muestreó durante todo el año y que ésta especie sólo se colectó en mayo, es posible que esta población realice movimientos estacionales y sea esta área donde se establece la colonia de maternidad. Los ejemplares se colectaron en las mismas condiciones que Myotis californicus.

\section{Corynorhinus townsendi australis Handley, 1955}

Ejemplares examinados, 1: $3 \mathrm{~km}$ NW San Pedro Actopan, $2400 \mathrm{~m}$ (ENCB).

Otros registros: 3 km NW San Pedro Actopan, 2400 m (Sánchez et al. 1989).

El único espécimen revisado es un macho, se capturó en septiembre y tenía una medida testicular de 11.0. Durante el periodo no reproductivo de la especie los machos tienden a ser solitarios (Barbour \& Davis 1969).

\section{Familia Molossidae}

\section{Tadarida brasiliensis mexicana (Saussure, 1860)}

Ejemplares examinados, 1: San Pedro Actopan, 2350 m (ENCB).

Este ejemplar es una hembra y fue colectado dentro de una vivienda. T. brasiliensis es una especie migratoria, por lo que es posible que no esté presente todo el año en la región. Para hábitats similares en el Estado de México se ha registrado en los meses fríos del año (González-Ruiz et al. 2004).

\section{Orden Xenarthra Familia Dasypodidae} Dasypus novemcinctus mexicanus Peters, 1864

No se capturó ningún ejemplar de esta especie, pero se encontraron dos excretas (probablemente de coyote) en la zona del volcán Pelagatos, con gran cantidad de placas óseas 
que pertenecían a individuos de esta especie. La presencia del armadillo en el área era de esperarse dado que se ha registrado en regiones cercanas como Zempoala (Davis \& Russell 1954, Ramírez-Pulido 1969) y Zoquiapan (Blanco et al. 1981).

\section{Orden Lagomorpha \\ Familia Leporidae \\ Romerolagus diazi (Díaz, 1893)}

Ejemplares examinados, 8: Volcán La Comalera, $3.5 \mathrm{~km}$ NE Chichinautzin (MZFC); $4.5 \mathrm{~km}$ S, $0.5 \mathrm{~km}$ W San Pablo Ostotepec (ENCB); Milpa Alta (IB-UNAM).

Otros registros: Volcán Tláloc (Velásquez et al. 1996); El Carril, delegación Milpa Alta, La Comalera, $3.2 \mathrm{~km}$ NE Chichinautzin (Ceballos \& Galindo 1984).

Todos los especimenes son hembras, colectadas en febrero, marzo, abril y noviembre. Tienen un peso medio de $555.7 \mathrm{~g}$ (478-649.5). El ejemplar de las cercanías de San Pablo Ostotepec se obtuvo después de haber sido cazado por un halcón cola roja (Buteo jamaicensis) dentro de un bosque de pino.

Sylvilagus floridanus orizabae (Merriam, 1893)

Ejemplares examinados, 1: $6.5 \mathrm{~km} \mathrm{~S}, 1 \mathrm{~km} \mathrm{~W}$ San Pablo Ostotepec (ENCB).

Además del ejemplar examinado, se observaron tres especimenes atropellados en las cercanías de San Pablo Ostotepec. Esta especie ha sido colectada en la misma región pero del lado de Morelos (Ramírez-Pulido 1969).

\section{Orden Rodentia Familia Sciuridae \\ Sciurus aureogaster nigrescens Bennett, 1833}

No se colectó ningún ejemplar de esta especie, pero se observaron dos individuos en el bosque de pino que se encuentra en los alrededores Santa Ana Tlacotenco y en la región del Tulmeac.

Spermophilus mexicanus mexicanus (Erxleven, 1777)

Ejemplares examinados, 1: $2 \mathrm{~km}$ antes del mirador la loma, carretera Xochimilco-Oaxtepec (UAMI).

Durante los recorridos que se hicieron por la región no se observó ningún ejemplar de ésta especie, sin embargo, los pobladores mencionan que la especie existe, pero que es escasa.

Spermophilus variegatus variegatus (Erxleben, 1777)

Ejemplares examinados, 3: $2 \mathrm{~km}$ antes mirador La Loma carretera Xochimilco Oaxtepec (UAMI); Milpa Alta (IB-UNAM).

Otros registros: Delegación Milpa Alta (sin localidad exacta; González-Romero 1981).

Además de los ejemplares examinados, una piel fue encontrada en una brecha a un costado del km 17 de la carretera Xochimilco-Oaxtepec. 
Familia Geomyidae

Thomomys umbrinus umbrinus (Richardson, 1829)

Ejemplares examinados: Ninguno.

Otros registros: Cicitepec, $15 \mathrm{~km} \mathrm{~S}, 5 \mathrm{~km}$ E Milpa Alta (Castro-Campillo \& Ramírez-Pulido 2000); Delegación Milpa Alta (sin localidad exacta; González-Romero 1981).

\section{Cratogeomys merriami merriami Thomas, 1893}

Ejemplares examinados, 2: Volcán Teutli, $3.2 \mathrm{~km} \mathrm{~N}, 1.2 \mathrm{~km} \mathrm{~W}$ Milpa Alta, $2550 \mathrm{~m}$ (ENCB). Uno de los ejemplares es una hembra capturada en junio dentro de un cultivo de maíz; no tenía signos aparentes de reproducción.

\section{Familia Heteromyidae}

\section{Liomys irroratus alleni (Coues, 1881)}

Ejemplares examinados, 7: Volcán Teutli, 1 km N, 2 km W Tecomitl, 2400 m (ENCB); Volcán Teutli, $3.2 \mathrm{~km} \mathrm{~N}, 1.2 \mathrm{~km} \mathrm{~W}$ Milpa Alta, $2550 \mathrm{~m}$ (ENCB); Volcán Teutli, $3.0 \mathrm{~km} \mathrm{~N}, 1.0 \mathrm{~km} \mathrm{~W}$ Milpa Alta, $2500 \mathrm{~m}$ (ENCB); $1 \mathrm{~km} \mathrm{S,} 3 \mathrm{~km}$ E Santa Ana Tlacotenco, $2650 \mathrm{~m}$ (ENCB). Otros registros: Delegación Milpa Alta (sin localidad exacta; González-Romero 1981).

Seis de los ejemplares son hembras adultas colectadas en agosto sin signos aparentes de actividad reproductiva. El único macho es un adulto que fue colectado en mayo con una longitud testicular de 25. Todos los ejemplares fueron capturados en áreas de pastizales con rocas, cerca de un bosque de encino y una zona de cultivos (Cuadro 1). Se colectó junto con Reithrodontomys fulvescens, $R$. megalotis, Peromyscus gratus, P. levipes, P. difficilis y Baiomys taylori.

\section{Familia Muridae}

\section{Reithrodontomys chrysopsis chrysopsis Merriam, 1900}

Ejemplares examinados, 15: $10 \mathrm{~km} \mathrm{~S}$ Santa Ana Tlacotenco, $2950 \mathrm{~m}$ (ENCB); La Quinta, 11 $\mathrm{km} \mathrm{S}, 2 \mathrm{~km}$ E Santa Ana Tlacotenco, $2850 \mathrm{~m}$ (ENCB); $9.5 \mathrm{~km} \mathrm{~S}, 2.5 \mathrm{~km}$ E Santa Ana Tlacotenco, $2850 \mathrm{~m}$ (ENCB).

Cinco hembras colectadas en mayo y junio tenían las glándulas lactógenas desarrolladas y una en mayo, se encontraba gestando tres embriones (11.0 mm cada uno). Siete machos capturados en febrero, mayo, julio y diciembre, tienen una longitud testicular media de 8.2 $\mathrm{mm}$ (5.0-9.0). Los ejemplares fueron capturados entre los pastos amacollados del bosque de pino y en la vegetación secundaria del bosque de oyamel. Otras especies colectadas junto a esta especie son Reithrodontomys megalotis, $R$. sumichrasti, Peromyscus difficilis, P. melanotis y Neotomodon alstoni.

\section{Reithrodontomys fulvescens toltecus Merriam, 1901}

Ejemplares examinados, 7: Volcán Teutli, 1 km N, 2 km W Tecomitl, 2400 m (ENCB); Volcán Teutli, $3.2 \mathrm{~km} \mathrm{~N}, 1.2 \mathrm{~km} \mathrm{~W}$ Milpa Alta, $2550 \mathrm{~m}$ (ENCB); Volcán Teutli, $3.0 \mathrm{~km} \mathrm{~N}, 1.0 \mathrm{~km} \mathrm{~W}$ Milpa Alta, $2500 \mathrm{~m}$ (ENCB); 4 km apróx. mirador la loma carretera Xochimilco-Oaxtepec (UAMI).

Otros registros: Delegación Milpa Alta (sin localidad exacta; González-Romero 1981). 
En enero, dos hembras no presentaban signos aparentes de reproducción y dos machos tenían una longitud testicular de $1.0 \mathrm{~mm}$ cada uno. Otro macho colectado en octubre tenía una longitud testicular de $12 \mathrm{~mm}$. Todos los ejemplares se colectaron asociados a zonas rocosas entre pastizal con Senecio y cultivos intercalados (Cuadro 1). También González-Romero (1981) encontró a esta especia entre los cultivos. En las mismas líneas de trampas también se colectó Liomys irroratus, Peromyscus difficilis, P. gratus, P. levipes y Baiomys taylori.

\section{Reithrodontomys megalotis saturatus Allen y Chapman (1897)}

Ejemplares examinados, 10: $1 \mathrm{~km} \mathrm{~S}, 3 \mathrm{~km}$ E Santa Ana Tlacotenco, $2650 \mathrm{~m}$ (ENCB); $9 \mathrm{~km}$ S, 4 km W San Salvador Cuahutenco, 3100 m (ENCB); La Quinta 11 km S, 2 km E Santa Ana Tlacotenco, $2850 \mathrm{~m}$ (ENCB).

Otros registros: Delegación Milpa Alta (sin localidad exacta; González-Romero 1981).

Seis hembras colectadas en marzo, abril y julio no tenían signos aparentes de reproducción. Cuatro machos colectados en julio tenían longitud testicular de $9.2 \mathrm{~mm}$ (9.5-10.0). Los ejemplares fueron capturados entre la vegetación secundaria del bosque de oyamel y entre el pastizal amacollado del bosque de pino, así como en el área rocosa.

\section{Reithrodontomys sumichrasti sumichrasti (Saussure, 1861)}

Ejemplares examinados, 2: La Quinta $11 \mathrm{~km} \mathrm{S,} 2 \mathrm{~km}$ E Santa Ana Tlacotenco, $2850 \mathrm{~m}$ (ENCB).

Los ejemplares son machos colectados en marzo y julio con medida testicular de 8.0 y 7.0 $\mathrm{mm}$. Las condiciones de captura fueron las mismas que para Reithrodontomys chrysopsis.

\section{Peromyscus difficilis (J. A. Allen, 1891)}

En la delegación Milpa Alta se registraron dos subespecies: P. difficilis amplus y P. difficilis felipensis. Las subespecies se distinguen principalmente por la coloración general del cuerpo (Hoffmeister \& De La Torre 1961), mientras que P. d. amplus presenta una coloración dorsal parda clara, en $P$. $d$. felipensis es parda oscura, casi negra. Estas dos subespecies tienen diferencias en la preferencia del hábitat $P$. $d$. amplus se asocia a la vegetación xerófila, y $P . d$. felipensis a regiones más altas en los bosque de coníferas.

\section{Peromyscus difficilis amplus Osgood, 1904}

Ejemplares examinados, 1: Volcán Teutli, $3.2 \mathrm{~km} \mathrm{~N}, 1.2 \mathrm{~km}$ W Milpa Alta, $2550 \mathrm{~m}$ (ENCB). Una hembra se colectó en enero sin signos aparentes de reproducción y fue capturada en las mismas condiciones que Liomys irroratus.

\section{Peromyscus difficilis felipensis Merriam, 1898}

Ejemplares examinados, 35: $9 \mathrm{~km} \mathrm{S,} 4 \mathrm{~km}$ W San Salvador Cuahutenco, $3100 \mathrm{~m}$ (ENCB); 10 km S Santa Ana Tlacotenco, 2950 m (ENCB); La Quinta, 11 km S, 2 km E Santa Ana Tlacotenco, $2850 \mathrm{~m}$ (ENCB); $4.5 \mathrm{~km} \mathrm{~S}, 3 \mathrm{~km}$ E Santa Ana Tlacotenco, $2800 \mathrm{~m}$ (ENCB). Otros registros: Delegación Milpa Alta (sin localidad exacta; González-Romero 1981). Una hembra colectada en febrero estaba gestando tres embriones de $5 \mathrm{~mm}$ de longitud cada uno. El resto de las hembras se capturaron en febrero, mayo, julio, noviembre y diciembre sin 
signos aparentes de reproducción. Veinte machos se encontraron en febrero, marzo, mayo, julio, noviembre con una longitud testicular media de $14.4 \mathrm{~mm}$ (5.0-26.0). Todos los especimenes se colectaron en áreas rocosas dentro de bosques de pino y oyamel. En las mismas líneas de trampas también se colectó a Reithrodontomys chrysopsis, $R$. megalotis, $R$. sumichrasti, Peromyscus melanotis, Neotomodon alstoni y Microtus mexicanus.

\section{Peromyscus gratus gratus Merriam (1898)}

Ejemplares examinados, 19: Volcán Teutli, $1 \mathrm{~km} \mathrm{~N}, 2 \mathrm{~km} \mathrm{~W}$ Tecomitl, $2400 \mathrm{~m}$ (ENCB); Volcán Teutli, $3.2 \mathrm{~km} \mathrm{~N}, 1.2 \mathrm{~km}$ W Milpa Alta, $2550 \mathrm{~m}$ (ENCB).

Las hembras fueron capturadas en enero y no presentan signos aparentes de reproducción. Los machos capturados en el mismo mes presentaron una longitud testicular media de $3.7 \mathrm{~mm}$ (2.0-6.0). Todos los ejemplares fueron colectados en las mismas condiciones que Peromyscus difficilis amplus.

\section{Peromyscus levipes levipes Merriam, 1898}

Ejemplares examinados, 1: Volcán Teutli, $3.2 \mathrm{~km} \mathrm{~N}, 1.2 \mathrm{~km}$ W Milpa Alta, $2550 \mathrm{~m}$ (ENCB). El ejemplar es un macho colectado en enero, con longitud testicular de $3.0 \mathrm{~mm}$. Fue capturado en las mismas condiciones que Peromyscus gratus y P. difficilis amplus.

\section{Peromyscus maniculatus (Wagner, 1845)}

En la delegación Milpa Alta se encuentran dos subespecies, Peromyscus maniculatus fulvus y $P$. m. labecula. Las diferencias que permiten la separación de estas dos subespecies son principalmente la coloración dorsal y el tamaño general del cráneo. En el caso de $P$. $m$. fulvus las tonalidades son más claras, las medidas craneales son relativamente más pequeñas y se encuentra asociado a la vegetación xerófila (Davis 1944). P. m. labecula tiende a tonalidades más obscuras, es mayor en tamaño y se asocia a regiones más altas y bosque de coníferas.

\section{Peromyscus maniculatus fulvus Osgood, 1904}

Ejemplares examinados, 3: Volcán Teutli, $3.2 \mathrm{~km} \mathrm{~N}, 1.2 \mathrm{~km} \mathrm{~W}$ Milpa Alta, $2550 \mathrm{~m}$ (ENCB); Volcán Teutli, $3.0 \mathrm{~km} \mathrm{~N}, 1.0 \mathrm{~km}$ W Milpa Alta, $2500 \mathrm{~m}$ (ENCB).

Otros registros: Milpa Alta, 2400 m (Hooper 1947).

\section{Peromyscus maniculatus labecula Elliot, 1903}

Ejemplares examinados, 1: $2 \mathrm{~km} \mathrm{~S}$ Santa Ana Tlacotenco $2860 \mathrm{~m}$ (ENCB).

El ejemplar fue el único capturado en una línea de trampas que se colocó en un bosque de encino, al borde del cráter del volcán San Miguel.

\section{Peromyscus melanotis J. A. Allen and Chapman, 1897}

Ejemplares examinados, 62: $4 \mathrm{~km}$ apróx. mirador la loma carretera Xochimilco Oaxtepec (UAMI); 7 km S, 3 km W San Salvador Cuahutenco, 3140 m (ENCB); 9 km S, 4 km W San Salvador Cuahutenco, 3100 m (ENCB); 10 km S Santa Ana Tlacotenco, $2950 \mathrm{~m}$ (ENCB); La Quinta, $11 \mathrm{~km} \mathrm{S,} 2 \mathrm{~km}$ E Santa Ana Tlacotenco, $2850 \mathrm{~m}$ (ENCB). Otros registros: Milpa Alta, $2 \mathrm{~km}$ del mirador La Loma, carretera Xochimilco-Oaxtepec; Milpa Alta, $5 \mathrm{~km}$ del Mirador La Loma, carretera Xochimilco-Oaxtepec (Martínez-Coronel et al. 1991). 
En la población de La Quinta se observó variación en la coloración dorsal en relación con la época del año. Los ejemplares de la temporada seca presentaron una coloración castaño ocre y en la temporada de lluvias castaño oscuro. Este mismo patrón en la coloración ya ha sido mencionado para la especie, pero en diferentes poblaciones del Eje Neovolcánico (Martínez-Coronel et al. 1991).

Los machos de febrero, marzo, mayo, julio, agosto y diciembre tenían una longitud promedio de los testículos de $10.3 \mathrm{~mm}$ (4.0-14.0), aunque no se encontró variación a través del año. De las 30 hembras colectadas, dos capturadas en marzo estaban gestando tres y cuatro embriones. Otras 11 hembras capturadas durante marzo, mayo y julio estaban lactando. Además, se encontraron 13 juveniles en febrero, mayo y julio. Según Davis (1944) esta especie se reproduce durante casi todo el año, sin embargo, en Milpa Alta se encontró que los periodos de gestación y crianza son sólo durante finales del invierno y toda la primavera. Los ejemplares fueron colectados en las mismas condiciones que Peromyscus difficilis felipensis.

Baiomys taylori analogous (Osgood, 1909).

Ejemplares examinados, 4: Volcán Teutli, $3.2 \mathrm{~km} \mathrm{~N}, 1.2 \mathrm{~km} \mathrm{~W}$ Milpa Alta, 2550 m (ENCB); $1 \mathrm{~km}$ E Tecomitl, $2240 \mathrm{~m}$ (ENCB).

Todos los ejemplares son hembras colectadas en enero y no poseen signos aparentes de reproducción. Esta especie se capturó tanto en la zona de cultivo como en pastizales. Junto con esta especie se colectó a Liomys irroratus, Reithrodontomys fulvescens, Peromyscus gratus, P. levipes y P. difficilis amplus.

Neotomodon alstoni Merriam, 1898

Ejemplares examinados, 20: $2 \mathrm{~km}$ antes mirador la loma carretera Xochimilco Oaxtepec (UAMI); 7 km S, 3 km W San Salvador Cuahutenco, 3140 m (ENCB); 9 km S, 4 km W San Salvador Cuahutenco, 3100 m (ENCB); $10 \mathrm{~km} \mathrm{~S} \mathrm{Santa} \mathrm{Ana} \mathrm{Tlacotenco,} 2950 \mathrm{~m}$ (ENCB); La Quinta, $11 \mathrm{~km} \mathrm{~S}, 2 \mathrm{~km}$ E Santa Ana Tlacotenco, $2850 \mathrm{~m}$ (ENCB); $4.5 \mathrm{~km} \mathrm{~S}, 3 \mathrm{~km}$ E Santa Ana Tlacotenco, $2800 \mathrm{~m}$ (ENCB). Otros registros: Delegación Milpa Alta (sin localidad exacta; González-Romero 1981).

Cuatro hembras colectadas en julio y agosto se encontraron lactando. Los machos se capturaron en los meses de febrero, mayo, julio y agosto, con una media testicular de 10.3 $\mathrm{mm}$ (8.0-14.0). El periodo de reproducción de esta especie es de marzo a agosto (Ceballos \& Galindo 1984), lo que coincide con lo encontrado en este estudio. Los ejemplares se colectaron asociados a pastizal amacollado en bosque abierto de pino. La fauna asociada a esta especie es la misma que la de Peromyscus melanotis.

\section{Neotoma mexicana torquata Ward, 1891}

Ejemplares examinados, 1: La Quinta, $11 \mathrm{~km} \mathrm{S,} 2 \mathrm{~km} \mathrm{E} \mathrm{Santa} \mathrm{Ana} \mathrm{Tlacotenco,} 2850 \mathrm{~m}$ (ENCB). Este espécimen fue encontrado muerto en una pileta. El lugar está rodeado de bosque de oyamel.

Microtus mexicanus mexicanus (Saussure, 1861)

Ejemplares examinados, 4: $2 \mathrm{~km}$ antes mirador la loma carretera Xochimilco Oaxtepec (UAMI); 4 km apróx. mirador la loma carretera Xochimilco Oaxtepec (UAMI); 7 km S, 3 km 
W San Salvador Cuahutenco, 3140 m (ENCB). También se encontraron molares asignables a esta especie en excretas de algunos carnívoros en el área del volcán Pelagatos, Cilcoayo y Tláloc, por lo que se infiere que su distribución incluye toda la parte sur de la Delegación.

En agosto se colectó una hembra gestante con dos embriones de $12.0 \mathrm{~mm}$ de longitud cada uno y un macho con longitud testicular de $7.0 \mathrm{~mm}$. Esta especie se reproduce durante todo el año, aunque con mayor frecuencia de mayo a agosto (Ceballos \& Galindo 1984).

\section{Orden Carnivora \\ Familia Canidae \\ Canis latrans cagottis (Hamilton-Smith, 1839)}

A pesar de que no se observó ningún ejemplar durante las visitas a la delegación Milpa Alta, en el volcán Pelagatos y Cilcuayo se escuchó el llamado característico de esta especie. Además se encontraron excretas presumiblemente de esta especie cerca del volcán Pelagatos que contenían restos de un armadillo (Dasypus novemcinctus). Los pobladores de la región mencionan que éste cánido todavía está presente en el área.

Urocyon cinereoargenteus nigrirostris (Linchtenstein, 1850)

Se observó un individuo que cruzaba la carretera Xochimilco-Oaxtepec a aproximadamente $12 \mathrm{~km}$ del límite con Morelos. González-Romero (1981) registra a la especie en San Juan Tezompa.

\section{Familia Mustelidae \\ Mustela frenata leucoparia (Merriam, 1896)}

En el km 37 de la carretera Xochimilco Oaxtepec fue encontrado un espécimen atropellado, pero desafortunadamente no se pudo recuperar los restos. También en la zona del volcán Cilcoayo, en San Salvador Cuahutenco y San Pablo Ostotepec se observaron otros ejemplares. Al parecer esta especie es relativamente abundante en la zona sur de la delegación Milpa Alta.

Spilogale putorius angustifrons Howell, 1902

Ejemplares examinados, 1: Volcán Pelagatos, 9 km S, 3.2 km E Santa Ana Tlacotenco, 2900 $\mathrm{m}$ (ENCB).

Sólo se encontró un cráneo y parte del esqueleto de un ejemplar en una pileta rodeada por bosque de pino.

\section{Mephitis macroura macroura Lichtenstein, 1832}

Ejemplares examinados, 1: $5 \mathrm{~km}$ NW Parrés, $2950 \mathrm{~m}$ (UAMI).

A pesar que la localidad del ejemplar examinado está referida a Parrés en la delegación Tlalpan, Distrito Federal, la localidad se ubica en la parte más oeste de Milpa Alta.

Conepatus mesoleucus mesoleucus (Linchtenstein, 1832)

Ejemplares examinados, 1: La Quinta, $11 \mathrm{~km} \mathrm{S,} 2 \mathrm{~km} \mathrm{E} \mathrm{Santa} \mathrm{Ana} \mathrm{Tlacotenco,} 2850 \mathrm{~m}$ (ENCB). 
Del ejemplar examinado sólo se tiene el cráneo sin la región occipital y fue encontrado cerca de una pileta que capta el agua de un manantial con bosque de oyamel a su alrededor. $C$. mesoleucus se ha registrado como muy abundante en la zona boscosa de Morelos (Davis \& Russell 1953, Álvarez-Castañeda \& López-Forment 1995).

\section{Familia Felidae}

Lynx rufus escuinapae J. A. Allen, 1903

Aunque no se colectó ni observó ésta especie, se incluye en este trabajo debido a que se han identificado huellas en Milpa Alta (Marcelo Aranda, com. pers.). Además, se tienen registros en Morelos muy cerca de los límites de la delegación (Álvarez-Castañeda 1996, Barrera 1954, Ramírez-Pulido 1969).

\section{Orden Artiodactyla \\ Familia Cervidae}

Odocoileus virginianus mexicanus (Gmelin, 1788)

En enero de 2000 se observó un ejemplar que llevaba un cazador cerca del área de La Quinta. Debido a la fuerte presión de cacería que se ejerce sobre esta especie, es muy probable que esté restringida a las zonas menos alteradas de las partes altas de la delegación.

\section{Especies con posible distribución en la Delegación de Milpa Alta.}

Con base en los registros que se tienen de la parte alta del norte de Morelos y sur del Estado de México, que colindan con el Distrito Federal y que comparten el mismo tipo de vegetación, se considera que en la delegación Milpa Alta es posible que se encuentren o hayan existido recientemente las siguientes especies: Sorex saussurei, Natalus stramineus, Lasiurus xanthinus, Sylvilagus cunicularius, Peromyscus hylocetes, Sigmodon leucotis, Bassariscus astutus, Taxidea taxus.

\section{DISCUSIÓN}

La historia geológica, ubicación geográfica y amplia variación altitudinal (2240$3300 \mathrm{~m}$ ) de la delegación Milpa Alta, le confiere una gran variedad de hábitats que se relaciona directamente con la diversidad de mamíferos. De esta manera, la cantidad de especies registradas para la delegación (45 especies) supera a la riqueza de especies de otras faunas regionales cercanas al Distrito Federal, como Zempoala (34 especies; Ramírez-Pulido 1969); Zoquiapan (38; Blanco et al. 1981) y el Ajusco (35; Aranda et al. 1980).

En la Cuenca de México se han registrado 87 especies (Ceballos \& Galindo 1984), de las cuales 76 también están presentes en el Distrito Federal (RamírezPulido et al. 1986, 2000). En la delegación Milpa Alta, están representadas el $54 \%$ de las especies que se encuentran en la Cuenca de México y el $61.8 \%$ de las del 
Distrito Federal, una riqueza relativamente alta si se toma en cuenta que la superficie de la Delegación es de sólo el 3.7\% de la Cuenca y el 19.2\% del Distrito Federal. El área de estudio alberga 2 géneros y 11 especies endémicas a México, la mayoría de estos taxa tienen su distribución restringida al Eje Neovolcánico Transversal (e. i. Neotomodon alstoni, Romerolagus diazi, Myotis volans amotus, Reithrodontomys chrysopsis chrysopsis, Thomomys umbrinus umbrinus y Cratogeomys merriami).

\section{Consideraciones para la conservación.}

La delegación puede dividirse en dos regiones para fines de conservación: la parte sur se encuentra incluida dentro de un corredor biológico semi-continuo que se extiende desde la región de Río Frío y entre los volcanes Iztacíhuatl y Popocatépetl hacia el oeste pasando por la Sierra del Chichinautzin, área del Ajusco, Sierra de Las Cruces, Zempoala, Sierras de Monte Alto y Monte Bajo. Todas estas serranías rodean el Distrito Federal y están incluidas en el Estado de México y Morelos. En la actualidad este corredor está muy fragmentado, ocasionando que la vegetación natural se encuentre repartida en islas (Fig. 1), lo que limita el flujo natural de las especies de mamíferos en el corredor, principalmente aquellas no voladoras que por sus características biológicas es poco probable que puedan salvar estas barreras ecológicas. Las especies endémicas como Peromyscus melanotis, Neotomodon alstoni y Romerolagus diazi y todos los Soricomorfos, son las más vulnerables al cambio del hábitat y al aislamiento (Veláquez et al. 1996), a pesar de esto todavía están presentes con distribución relativamente amplia, pero se desconoce el impacto de la fragmentación sobre sus poblaciones.

La parte norte de la Delegación corresponde al sur de la Cuenca de México, desafortunadamente está severamente alterada por los campos agrícolas y la urbanización (Fig. 1); se ha estimado que poco más de $95 \%$ de la superficie ha sido afectada por alguna actividad antropogénica (Navarro-Frías, obs pers.). Además, esta región se encuentra aislada del resto de la Cuenca debido a la mancha urbana que la rodea. Este aislamiento limita la migración y el flujo génico de las poblaciones de mamíferos hacia el norte de la Cuenca y es muy propensa al impacto negativo de la urbanización de la Ciudad de México. Estas alteraciones ya han tenido sus consecuencias, pues es posible que especies con distribución potencial en esta parte de la delegación, ya estén extirpadas (e. i. Dipodomys phillipsii, Spermophilus mexicanus y Lynx rufus).

La influencia y el impacto negativo que la Ciudad de México puede tener sobre áreas aún conservadas de la Cuenca de México, son muy altos pero aún no han sido evaluados en detalle. Sin embargo, el futuro de estas regiones no es alentador, si tomamos en cuenta el efecto adverso reciente de la mancha urbana sobre la composición y diversidad de la fauna silvestre en otras áreas del Distrito Federal, como en Santa Catarina (Castro-Campillo et al. 1992) y en otras partes de la Cuenca de México (Ceballos \& Galindo 1984). En la última década se han hecho diferentes 
propuestas para crear áreas prioritarias para la conservación (Caldecott et al. 1996); estas propuestas contemplan áreas con mayor cantidad de especies, grupos de especies amenazados y vulnerables (Ceballos et al. 2002). La región sur de la delegación Milpa Alta tiene varias cualidades que cumplen estas condiciones: (1) la zona es muy rica en especies y es mayor que otras áreas aledañas, (2) contiene un número considerable de especies vulnerables (e. i. Lynx rufus, Odocoileus virginianus y Romerolagus diazi), (3) la décima parte de los géneros y una cuarta parte de las especies de la delegación son endémicas a México, (4) la zona es parte del corredor biológico del centro del Eje Volcánico Trasversal, (5) es refugio temporal de especies migratorias (Leptonycteris yerbabuenae y Tadarida brasiliensis) y especies con movimientos altitudinales (e. i. Lasiurus), y (6) el área total presenta una baja alteración de la vegetación original (67\% de su superficie; INEGI, 1997). De acuerdo con lo anterior, la parte sur de la delegación Milpa Alta debe considerarse como unos de los refugios más importantes para la conservación de la mastofauna en la Cuenca de México y del Distrito Federal, y es en esta región donde deben enfocarse los programas para la conservación de la mastofauna.

\section{AGRADECIMIENTOS}

Nuestra gratitud al Dr. Fernando Cervantes del Instituto de Biología de la Universidad Nacional Autónoma de México (IBUNAM), a la M. en C. Livia León del Museo de Zoología "Alfonso L. Herrera" de la Facultad de Ciencias de la Universidad Nacional Autónoma de México (MZFC), al Dr. José Ramírez Pulido de la Universidad Autónoma Metropolitana Unidad Iztapalapa (UAMI) y al M. en C. Juan Carlos López Vidal de Escuela Nacional de Ciencias Biológicas del Instituto Politécnico Nacional (ENCB) por permitirnos examinar el material de las colecciones a su cargo. Un sincero reconocimiento a Aurelio Ocaña, Jorge Villalpando y Nansy Sánchez por la preparación de la mayoría del material colectado y en especial a Gabriel Villegas, Cuahutémoc Nieto, Miguel Blancas y Nashieli Jau que desinteresadamente nos brindaron su apoyo durante el trabajo de campo. A Noemi Matías y dos revisores anónimos quienes con sus comentarios y observaciones ayudaron a mejorar este trabajo.

\section{LITERATURA CITADA}

Álvarez, T. \& S. T. Álvarez-Castañeda. 1996. Aspectos biológicos y ecológicos de los murciélagos de Ixtapan del Oro, Estado de México, México. Pp. 169-182 In: H. H. Genoways y R. J. Baker (Eds.). Contributions in Mammalogy: A Memorial Volume Honoring Dr. J. K. Jones, Jr. Museum Texas Tech University. Lubbock, Texas.

Álvarez, T., S. T. Álvarez-Castañeda \& J. C. López-Vidal. 1994. Claves para murciélagos mexicanos. Centro de Investigaciones Biológicas del Noroeste, S. C. y Escuela Nacional de Ciencias Biológicas, Instituto Politécnico Nacional, México D. F.

Álvarez-Castañeda, S. T. 1996. Los mamíferos del estado de Morelos. Centro de Investigaciones Biológicas del Noroeste. S. C. 211 pp. 
Álvarez-Castañeda, S. T., \& W. López-Forment. 1995. Datos sobre los mamíferos del área cercana a Palpan, Morelos. Anal. Inst. Biol., UNAM 66:123133.

Aranda, S., C. Martínez del Río, L. del C. Colmenero \& V. M. Magallón. 1980. Los Mamíferos de la Sierra del Ajusco. Comisión Coordinadora para el Desarrollo Agropecuario del Distrito Federal, 146 pp.

Arita, T. H. \& S. R. Humphrey. 1988. Revisión taxonómica de los murciélagos magueyeros del género Leptonycteris (Chiroptera: Phyllostomidae). Acta Zool. Mex. (n.s.) 29:1-60.

Baker, R. H. \& C. J. Phillips. 1965. Mammals from El Nevado de Colima, Mexico. J. Mammal. 46:691-693.

Barbour, R. W. \& W. H. Davis. 1969. Bats of America. Univ. Press, Kentucky, Lexington, 286 pp.

Barrera, A. 1954. Notas sobre sifonápteros. VII. Lista de especies colectadas en el Municipio de Huitzilac, Morelos y diagnosis de Pleochaetis apollinaris aztecus subsp. nov. (Ins. Siph.). Ciencias, México 14:137-139.

Blanco, Z. S., G. Ceballos, C. L. Galindo, J. M. Maass, R. Patrón, A. Pescador \& A. I. Suárez, 1981. Ecología de la estación experimental Zoquiapan: descripción general, vegetación y fauna. Universidad Autónoma de Chapingo, Colección Cuadernos Universitarios No. 2, Serie Agronomía, Chapingo, Estado de México. 114 pp.

Bogan, M. A. 1999. Family Vespertilionidae. Pp. 139-181. In: S. T. Álvarez-Castañeda y J. L. Patton (Eds.). Mamiferos del Noroeste de México. Centro de Investigaciones Biológicas del Noroeste, S. C. La Paz.

Caldecott, J. O., M. D. Jenkins, T. H. Johnson \& B. Groombrige. 1996. Priorities for conserving global species richness and endemism. Biodivers. and Conserv. 5:699-727.

Castro-Campillo, A., B. A. Silva T. \& J. Ramírez-Pulido. 1992. Notas sobre los mamíferos de la Sierra de Santa Catarina, Distrito Federal, México. Rev. Soc. Mex. Hist. Nat. 43:33-42.

Castro-Campillo, A. \& J. Ramírez-Pulido. 2000. Systematics of the smoth-toothed pocket gopher, Thomomys umbrinus, in the Mexican Transvolcanic Belt. Amer. Mus. Nat. Hist., Novitates 3297:1-37.

Ceballos, G., J. Arroyo-Cabrales \& R. A. Medellín. 2002. Mamíferos de México. Pp. 377413. In: G. Ceballos y J. A. Simonetti (Eds.). Diversidad y Conservación de los Mamiferos Neotropicales. CONABIO-UNAM. México, D. F.

Ceballos, G. \& C. Galindo. 1984. Mamíferos silvestres de la Cuenca de México. Limusa, México D. F.

Davis, W. B. 1944. Notes on Mexican Mammals. J. Mammal. 25:370-403.

Davis, W. B. \& R. J. Russell. 1953. Aves y mamíferos del Estado de Morelos. Rev. Soc. Mex. Hist. Nat. 14:77-147. 1954. Mammals of the Mexican state of Morelos. J. Mammal. 35:63-80.

Fitch, J. H., K. A. Shump, Jr., \& A. U. Shump. 1981. Myotis velifer. Mammal. Species 149:1-5.

Guerrero-Enríquez, J.A., M. de L. Romero-Almaraz y C. Sánchez-Hernández. 1996. Myotis volans amotus in Morelos, Mexico. Bat Research News 37:41.

González-Romero, A. 1981. Roedores plaga en las zonas agrícolas del Distrito Federal. Publicación No. 7. Instituto de Ecología, Museo de Historia Natural de la Ciudad de México. México, D. F. 
González-Ruiz, N., F. Navarro-Frías, T. Álvarez, \& S. T. Álvarez-Castañeda. 2004. Los murciélagos del estado de México en la colección de mamíferos de la Escuela Nacional de Ciencias Biológicas, I. P. N. Pp. 53-82. In: A. Castro Campillo y J. Ortega (Eds.). Homenaje a la trayectoria Mastozoológica de José Ramírez Pulido. Universidad Autónoma Metropolitana, Iztapalapa, México, D. F.

Hall, E. R. 1981. The mammals of North America. 2da. ed. Vol. 1 y 2, John Wiley and Sons, New York.

Hall, E. R., \& B. Villa R. 1949. An annotated check list of the mammals of Michoacan, Mexico. Univ. Kansas Pub., Mus. Nat. Hist. 1: 431-472.

Hennings, D. \& R. S. Hoffmann. 1977. A review of the taxonomy of the Sorex vagrans species complex from western North America. Occ. Pap. Univ. Kansas, Mus. Nat. Hist. 68:1-35.

Hoffmeister, D. F. \& De La Torre. 1961. Geographic variation in the mouse Peromyscus difficilis. J. Mammal. 42:1-13.

Hooper, E. T. 1947. Notes on Mexican mammals. J. Mammal. 28:40-57.

1952. A systematic review of the harvest mice (genus Reithrodontomys) of Latin America. Misc. Pub. Mus. Zool., Univ. Michigan 77: 1-225.

INEGI, 1997. Cuaderno estadístico delegacional. Milpa Alta. México, D. F.

Martínez-Coronel, M., J. Ramírez-Pulido \& T. Álvarez. 1991. Variación intrapoblacional e interpoblacional de Peromyscus melanotis (Rodentia: Muridae) en el Eje Volcánico Transverso, México. Acta Zool. Mex. (n.s.) 47:1-51.

Miller, G. S., Jr. \& G. M. Allen. 1928. The American bats of the genera Myotis volans and Pizonyx. Bull. U. S. Nat. Mus. 144:1-218.

Ramírez-Pulido, J. 1969. Contribución al estudio de los mamíferos del Parque Nacional Lagunas de Zempoala, Morelos, México. Anal. Inst. Biol. UNAM, Serie Zoología 40:252290.

Ramírez-Pulido, J. \& T. Álvarez. 1972. Notas sobre los murciélagos del género Leptonycteris en México, con la designación del lectotipo de L. yerbabuenae Martínez y Villa, 1940. Southwest. Nat. 16:249-259.

Ramírez-Pulido, J. Arroyo-Cabrales \& A. Castro-Campillo. 2005. Estado actual y relación nomenclatural de los Mamíferos terrestres de México. Acta Zool. Mex. (n.s.) 21:21-82.

Ramírez-Pulido, J., J. M. C. Britton, A. Perdomo \& A. Castro. 1986. Guía de los mamiferos de México. Referencias hasta 1983. Universidad Autónoma Metropolitana Unidad Iztapalapa, México, D.F.

Ramírez-Pulido, J., A. Castro-Campillo, M. A. Armella \& A. Salame-Méndez. 2000. Bibliografia reciente de los mamíferos de México: 1994-2000. Universidad Autónoma Metropolitana, Unidad Iztapalapa, México, D.F.

Ramírez-Pulido, J., G. Ceballos \& S. L. Williams. 1980. A noteworthy record of the longlegged Myotis (Myotis volans) from central Mexico. Southwest. Nat. 25:124.

Reyes, A. 1989. Milpa Alta, monografía. Comisión Coordinadora para el Desarrollo Agropecuario del Distrito Federal, México, D.F. 
Rzedowski, J. 1975. Flora y Vegetación en la Cuenca de México. Págs 79-134 In: Memorias de las Obras del Sistema de Drenaje Profundo del Distrito Federal. Departamento del Distrito Federal. México, D. F. Tomo 1.

Rzedowski, J. \& G. C. Rzedowski. 1979. Flora Fanerogámica del Valle de México. Vol. I Editorial C.E.C.S.A., México D. F.

Sánchez, O., G. Lopez-Ortega \& R. Lopez-Wilchis. 1989. Murciélagos de la Ciudad de México y sus alrededores. Pp. 141-165. In: R. Gío-Argáez, I. Hernández-Ruíz y E. SáinzHernández (Comps.). Ecología Urbana. Sociedad Mexicana de Historia Natural, Publicación Especial, México, D.F.

SEMARNAT. 2002. Norma Oficial Mexicana NOM-059-ECOL—2002, que determina las especies y subspecies de flora y fauna Silvestre terrestre y acuática en peligro de extinción, amenazadas, raras y sujetas a protección especial, y establece especificaciones para su protección. Diario Oficial de la Federación. 6 de marzo de 2002. México D. F.

Shump, K. A., Jr. \& A. U. Shump. 1982a. Lasiurus borealis. Mammal. Species 183:1-6. . 1982b. Lasiurus cinereus. Mammal. Species 185:1-5.

Velázquez, A., F. J. Romero \& L. León. 1996. Fragmentación del hábitat del conejo zacatuche. Pp. 73-86. In: Ecología y conservación del conejo zacatuche y su hábitat. A. Velázquez, F. J. Romero y J. López-Paniagua (Comps.). Ediciones Científicas Universitarias, México, 205 pp.

Villa-Ramírez, B. 1953. Mamíferos silvestres del Valle de México. Anal. Inst. Biol., UNAM 23:269-492.

1967. Los murciélagos de México. Su importancia en la economía y la salubridadSu clasificación sistemática. Instituto de Biología, Universidad Nacional Autónoma de México. México, D. F.

Villalpando, J. A. \& T. Álvarez. 2000. Murciélagos (Chiroptera) de Michoacán en la colección de mamíferos de la Escuela Nacional de Ciencias Biológicas del IPN, México. Anal. Esc. Nac. Cienc. Biol., IPN, 46:119-188.

Watkins, L. C., J. K. Jones, Jr. \& H. H. Genoways. 1972. Bats of Jalisco, Mexico. Spec. Pub. The Museum, Texas Tech Univ. 1:1-44.

Webster, D. W. 1993. Systematics and evolution of bats of genus Glossophaga. Spec. Pub. The Museum, Texas Tech Univ. 1:1-184.

Recibido: 11 de enero de 2006

Aceptado: 12 de junio de 2007 